BEHAVIOUR

\title{
Gender differences in sexual behaviours in response to genitourinary symptoms
}

\author{
A Khan, J D Fortenberry, M' H Temkit, W Tu, D P Orr, B E Batteiger
}

Sex Transm Infect 2005;81:262-266. doi: 10.1136/sti.2004.010587

See end of article for authors' affiliations

....................

Correspondence to: Ayesha Khan, MD, Division of Infectious Diseases, Indiana University School of Medicine, 545 Barnhill Drive, EH Room 435 Indianapolis, IN 46202, USA; ayeskhan@iupui.edu

Accepted for publication 15 September 2004

\begin{abstract}
Objective: To understand gender differences in sexual behaviours in response to genitourinary symptoms. Methods: 473 (239 female and 234 male) subjects were enrolled at an STD clinic regardless of symptoms or infection status. Subjects completed a 30 day calendar recall interview of genitourinary symptoms, coital activity, sexual partners, and condom use.

Results: Of the total of 473 participants, 261 (55\%) reported symptoms (61\% women and 39\% men). STI prevalence was $73 \%$ and $75 \%$ for symptomatic women and men, respectively. For black women the probability of coitus was decreased in the presence of vaginal discharge (OR $0.64,95 \% \mathrm{Cl} 0.47$ to 0.89 ). No change in coital activity was seen in non-black women in the presence of vaginal discharge. Having vaginal discharge did increase the likelihood of condom use by their partners $(O R 2.48,95 \% \mathrm{Cl} 1.05$ to 5.88), if coitus occurred. Urethral discharge was not associated with coitus or condom use in men. However, in men, dysuria was associated with increased likelihood of condom use (OR 4.25, 95\% Cl 1.57 to 11.56 ) if coitus occurred.

Conclusion: Black women altered both coital activity and condom use behaviours in response to vaginal discharge. In contrast, non-black women did not modify coital activity. Men increased condom use when having dysuria but did not alter coital activity. Changes in sexual behaviours may alter the risk of STI transmission independent of interactions with the healthcare system. STI education and prevention programmes need to better understand these gender and racial differences in developing effective strategies to reduce STI transmission.
\end{abstract}

G enitourinary symptoms are an important stimulus to seek health care related to concern for sexually transmitted infections (STI) or by causing physical discomfort. Even before seeking care, symptomatic individuals may modify their sexual behaviours based on previous experiences, prevalent cultural beliefs, and perceptions of normal versus abnormal health status. ${ }^{12}$ However, individuals with STI related symptoms frequently fail to take protective action through prompt care seeking or curtailing sexual activity, and using condoms when symptomatic. ${ }^{34}$ People who are infected can reduce transmission to others by early treatment, reducing sexual activity, and using condoms.

Gender differences in response to genitourinary symptoms are important since sexual activity and prophylactic behaviours such as condom use after an STI is established are critical determinants of disease transmission. ${ }^{5}$ In one study $40 \%$ of respondents continued to have sex while symptomatic. Though a lower percentage of men (34\% v 50\%) than women reported coital activity while symptomatic, approximately one third of these men had two or more partners. ${ }^{4}$ Women in general, particularly married women and those in established relationships, were more likely to have sex while symptomatic. ${ }^{6}$

These gender differences have implications for clinical and public health prevention programmes. Prevailing social norms, racial origin ${ }^{257}$ and biological differences affect the way women and men respond when they have STI symptoms. $^{89}$ In this study we examined the effect of genitourinary symptoms, by gender and race, on sexual behaviours using a detailed 30 day daily calendar recall method. Previous studies have looked at STI related symptoms and sexual behaviours using retrospective recall of average behaviours after symptom onset. This approach may enhance recall error or socially desirable responses. ${ }^{10-12}$ In our study we used the 30 day calendar recall method that has been shown to have acceptable reliability and accuracy. ${ }^{11}{ }^{12}$ Using the calendar recall questionnaire we sought to identify the relation between STI symptoms and gender differences in sexual behaviours.

\section{METHODS}

\section{Settings and enrolment}

Data for this analysis were taken from an ongoing study designed to evaluate STI risks and protective factors associated with the transmission of bacterial and protozoan STI in the context of sexual partnerships. Although the overall study is focused on analysis of sexual partnerships the current study takes into account all eligible participants regardless of whether their partners were enrolled.

Subjects between the ages of 15-24 years (regardless of genital symptoms or infection status) visiting a county STD clinic in Indianapolis, Indiana, were invited to participate. Only the initial clinic visit was considered in this analysis for index subjects and their partners. Eligibility criteria included sexual activity in the past 30 days, willingness to identify all sexual partners in the past 30 days and a working knowledge of English. Exclusion criteria were antibiotic use in the past 30 days, known HIV infection or any other immune suppressive condition, clinic visit necessitated by sexual assault, and emotional or mental health conditions that would preclude partner enrolment. The most common reasons for non-participation were lack of time and lack of interest.

Partners, aged 14 or older, of the index subjects were recruited through the initial clinic visit or contacted and enrolled by the research disease intervention specialists. Informed consent was obtained from all eligible participants.

Abbreviations: BV, bacterial vaginosis; NGU, non-gonococcal urethritis; STI, sexually transmitted infections 


\begin{tabular}{|c|c|c|}
\hline \multirow[b]{2}{*}{ Variables } & \multirow{2}{*}{$\begin{array}{l}\text { Women } \\
(n=239)\end{array}$} & \multirow{2}{*}{$\begin{array}{l}\text { Men } \\
(n=234)\end{array}$} \\
\hline & & \\
\hline Age, mean (SD) & $20(3.4)$ & $22(4.5)$ \\
\hline \multicolumn{3}{|l|}{ Race $^{*}$ (n) } \\
\hline African-American, $n(\%)$ & $149(62)$ & $181(77)$ \\
\hline White, $n(\%)$ & $82(34)$ & $46(20)$ \\
\hline Age at first sex, mean (SD) & $14.6(2.0)$ & $13.5(2.7)$ \\
\hline Lifetime partners, median (range)* & $8(1-50)$ & $15(1-100)$ \\
\hline Partners in 30 days, median (range) ${ }^{*}$ & $1(1-8)$ & $1(1-12)$ \\
\hline Previous sexually transmitted infection, $n(\%)$ & $152(63)$ & $129(55)$ \\
\hline High school/GED or less, n (\%)* & $173(73)$ & $188(81)$ \\
\hline Unemployed, $\mathrm{n}(\%)$ & $75(31)$ & $82(35)$ \\
\hline Index subjects, $\mathrm{n}(\%)$ & $176(74)$ & $165(71)$ \\
\hline Partners, $\mathrm{n}(\%)$ & $63(26)$ & $69(29)$ \\
\hline Symptom days $<7$ to care seeking, $n(\%)^{*}$ & $58(37)$ & $69(68)$ \\
\hline$>7$ days to care seeking, $n(\%)^{*}$ & $100(63)$ & 34 (33) \\
\hline Symptomatic, $n(\%)^{*}$ & $158(66)$ & $103(44)$ \\
\hline Discharge, $\mathrm{n}(\%)$ & $114(72)$ & 78 (76) \\
\hline Dysuria, n $(\%)^{*}$ & $40(25)$ & $64(62)$ \\
\hline Abdominal/pelvic pain & $73(46)$ & - \\
\hline
\end{tabular}

The study protocol was reviewed and approved by the Indiana University-Purdue University Indianapolis institutional review board.

\section{Data collection}

Subjects completed an enrolment questionnaire to provide demographic information such as age, race, gender, education, reasons for seeking care, age at first sexual intercourse, previous STIs, and total number of partners in the past 30 days. Interviews were conducted by trained research staff using a 30 day calendar recall and memory cues for coitus and the presence of genitourinary symptoms. Participants were asked to identify each day on which coitus occurred during the previous 30 days. For each day with coital activity, participants were asked about the number of sexual partners, the number of coital events with each partner, and whether a condom was used during each identified coital event.

Three genitourinary symptoms (vaginal discharge, dysuria, and lower abdominal/pelvic pain) for women and two symptoms for men (urethral discharge and dysuria) were sought using the 30 day calendar recall. Subjects were considered asymptomatic if no symptoms were reported for the preceding 30 days.

\section{Laboratory data}

Specimens were collected by experienced clinical or research staff and processing was carried out at Indiana University laboratory facilities. Subjects underwent Neiserria gonorrhoeae and Chlamydia trachomatis culture testing on cervical (women) and urethral (men) specimens. From women, rectal and urethral swabs were obtained for $N$ gonorrhoeae and $C$ trachomatis cultures, respectively. PCR testing for $N$ gonorrhoeae and C trachomatis was done on cervical, vaginal, urethral, and urine specimens for women and on urethral and urine specimens for the men using Cobas Amplicor CT/ NG (Roche Diagnostic Systems, Indianapolis, IN, USA). PCR testing for $T$ vaginalis was done on vaginal (women) and urine (women and men) specimens. The $T$ vaginalis $P C R$ test was based on an Amplicor platform with primers specific for $T$ vaginalis as previously described. ${ }^{13}$ For the purpose of our analysis a subject was considered infected if at least one test specimen was positive.

Women also underwent wet mount and $\mathrm{KOH}$ slide examinations for the diagnosis of bacterial vaginosis (BV) and vaginal candidiasis. The clinical criteria used for diagnosis of BV are (1) homogeneous, greyish white discharge, (2) a positive whiff test, (3) presence of clue cells, (4) vaginal $\mathrm{pH}>4$.5. Candidiasis was diagnosed on the basis of clinical presentation with thick whitish discharge and visualisation of yeast forms or hyphae on microscopy using $\mathrm{KOH}$ preparation. For the purpose of our analysis, chlamydia negative, non-gonococcal urethritis (NGU) in men was defined as five or more white blood cells per high power field on Gram stain of endourethral secretions and negative tests for gonorrhoea, chlamydia, and trichomonas.

\section{Data analysis}

The demographic and clinical characteristics of women and men were reported separately in a tabular form. Comparisons between the two sexes were performed using $t$ test and $\chi^{2}$ test for continuous and discrete variables, respectively. To examine the effect of symptoms on sexual behaviours in symptomatic subjects, we used logistic regression models with random effects. In this analysis, because each individual had contributed 30 daily records on coitus and condom use in his or her recall interview, we used a random subject effect to account for the within subject correlation among the repeated measures from the same individual. Variables included in the initial models were age, race, symptoms, symptom duration, education, previous STIs, employment status, and marital status. However, in the final models only significant variables were analysed. Women and men were modelled separately to accommodate the gender specific symptoms. The outcome variables were coital activity and condom use in symptomatic women and men. Models were fitted using SAS PROC NLMIXED. Odds ratios and 95\% confidence intervals of the odds ratios were used to summarise the effects of the variables in the models.

\section{RESULTS}

A total of 473 subjects ( 239 women and 234 men) were enrolled from April 2000 through August 2002. The mean age was 21 years (range 14-39 years), the majority were AfricanAmerican, and 27\% were white. Approximately two thirds reported having an STI in the past and one third were unemployed (table 1).

In all, $212(45 \%)$ subjects reported no symptoms in the preceding 30 days and were considered asymptomatic. Of the $261(55 \%)$ symptomatic subjects who reported one or more symptoms on any given day before the clinic visit, 158 (61\%) were women and 103 (39\%) were men. Genital discharge was the most common symptom reported by both symptomatic 


\begin{tabular}{|c|c|c|}
\hline \multirow[b]{2}{*}{ Symptom† } & \multirow{2}{*}{$\begin{array}{l}\text { Coital activity } \\
\text { OR }(95 \% \mathrm{Cl})\end{array}$} & \multirow{2}{*}{$\begin{array}{l}\text { Condom use } \\
\text { OR }(95 \% \mathrm{Cl})\end{array}$} \\
\hline & & \\
\hline $\begin{array}{l}\text { Vaginal discharge } \\
\text { Black women } \\
\text { Non-black women } \\
\text { Abdominal/pelvic pain } \\
\text { Dysuria }\end{array}$ & $\begin{array}{l}0.80(0.70 \text { to } 0.90) \\
0.64(0.47 \text { to } 0.89) \\
1.13(0.75 \text { to } 1.70) \\
1.20(0.89 \text { to } 1.62) \\
0.86(0.56 \text { to } 1.34)\end{array}$ & $\begin{array}{l}2.48(1.05 \text { to } 5.88) \neq \\
- \\
- \\
0.72(0.26 \text { to } 1.99) \\
2.65(0.72 \text { to } 9.78)\end{array}$ \\
\hline \multicolumn{3}{|c|}{$\begin{array}{l}\text { *Sample includes only symptomatic women }(n=158) \text {. } \\
\text { †Vaginal discharge }(n=114) \text {, dysuria }(n=40) \text {, and abdominal/pelvic pain }(n=73) \text {. The sum total is greater thar } \\
158 \text { because some women reported more than one symptom. } \\
\text { łlnteraction between race and condom use not significant. }\end{array}$} \\
\hline
\end{tabular}

women $(72 \%)$ and men $(76 \%)$. For both sexes, symptoms always persisted after the initial report.

From the total of 473 subjects, there were 341 index subjects $(72 \%)$ and 132 partners (28\%). In our analysis of index subjects and partners as two possibly separate groups, we did not find any significant differences in overall symptom prevalence or a greater proportion of asymptomatic individuals in the partner group (data not shown). Therefore, for the purpose of our study we analysed index subjects and partners as a single group.

\section{Genitourinary symptoms and STIs}

STI prevalence was $73 \%$ in the symptomatic group compared to the $50 \%$ in the asymptomatic group (OR 1.5, $95 \%$ CI 1.10 to 3.84$)$. However, in the asymptomatic group $63 \%$ women had an STI, BV, or vaginal candidiasis compared to $42 \%$ men (OR 1.6, 95\% CI 1.33 to 6.75). When adjusted for the presence of BV and vaginal candidiasis in women this difference in infection prevalence became statistically insignificant.

Using the calendar recall questionnaire 261 (55\%) of the subjects reported symptoms on at least 1 day. However, only $64(13 \%)$ considered genital symptoms as the main reason for the clinic visit on the enrolment questionnaire. Commonly cited reasons for seeking care were concerns for STI, desire for screening, and sexual partner enrolment. Women were less likely than men to report symptoms as the main reason for seeking care (data not shown).

\section{Genitourinary symptoms and sexual behaviours}

Multivariable logistic models were used to study the relation between genitourinary symptoms and sexual behaviours in women and men adjusting for race (blacks and non-blacks). We examined the effects of individual genitourinary symptoms on coital activity and condom use without adjusting for the presence of other symptoms. For black women in contrast with non-black women (table 2), the presence of vaginal discharge was associated with a decreased likelihood of coitus on any given day (OR $0.64,95 \%$ CI 0.47 to 0.89 ). For both black and non-black women, vaginal discharge increased the likelihood of condom use by their partners (OR 2.48, 95\% CI 1.05 to 5.88). Abdominal/pelvic pain and dysuria in women did not seem to have any significant effect on coital activity or condom use.

For men, in the presence of dysuria condom use was increased (OR 4.25, 95\% CI 1.57 to 11.56). Dysuria did not have any significant associations with coital activity. The multivariable logistic model did not show any association of urethral discharge on coital activity or condom use (table 3 ).

\section{DISCUSSION}

In our study women reduced coital activity in the presence of vaginal discharge and reported increased condom use by their partners. After adjusting for race black women having vaginal discharge were more likely to reduce coital activity than non-black women. These are interesting racial differences among women in response to vaginal discharge. There could be several explanations for why black women reduced coital activity than non-black women, when having vaginal discharge. Possible reasons for reducing coital activity may be a greater emphasis on personal hygiene ${ }^{14}$ or a better awareness of symptoms and their consequences among black women. Evans et al reported in two studies that racial origin is independently linked to sexual behaviours and the incidence of STIs. ${ }^{15}$ It is possible that the overall higher prevalence of STIs in the black community could result in greater awareness of symptoms and promote preventive sexual behaviours among black women. ${ }^{15}$ It is unclear whether being informed of an STI prompts people to change their sexual behaviour. However, Warszawski et al showed that in women, a previous episode of STI was associated with risk reduction in sexual behaviours. ${ }^{16}$

In contrast with our study, other studies have shown that women generally continue sexual activity while symptomatic with a steady partner or spouse. ${ }^{4}{ }^{6}$ These women may be unable to refuse sex or insist on condom use with long term partners, especially if such a request implies a partner being unfaithful, not committed to the relationship, or resisting condom use. ${ }^{17}{ }^{18}$ Our study did not address the relationship duration or partner type, both of which are well known factors that influence coital activity and condom use. ${ }^{17-21}$ Additionally, our study population included mainly single

Table 3 Multivariate analysis of genitourinary symptoms on sexual behaviours of men*

\begin{tabular}{|c|c|c|}
\hline & Coital activity & Condom use \\
\hline Symptom† & OR $(95 \% \mathrm{Cl})$ & OR $(95 \% \mathrm{Cl})$ \\
\hline $\begin{array}{l}\text { Urethral discharge } \\
\text { Dysuria }\end{array}$ & $\begin{array}{l}0.92(0.66 \text { to } 1.29) \\
0.92(0.63 \text { to } 1.36)\end{array}$ & $\begin{array}{l}2.04(0.87 \text { to } 4.78) \\
4.25(1.57 \text { to } 11.56\end{array}$ \\
\hline
\end{tabular}


women who might be more sexually assertive, without the same marital or social obligations.

STI transmission is dependent both on the length of time an individual remains infected and the frequency of unprotected coital activity that occurs during this period. ${ }^{522}$ In our study women with vaginal discharge reported increased condom use by their partners. This protective modification in sexual behaviour before any interaction with the healthcare system may be an effective strategy in interrupting STI transmission from women to their male partners but increase the women's risk of complications especially if health care is not promptly sought.

Our study did not show any significant effect of abdominal/pelvic pain or dysuria on coital activity and condom use in women. Though not reaching statistical significance the presence of dysuria among women appears to increase the likelihood of condom use in their partners (OR $2.65,95 \%$ CI 0.72 to 9.78 ). It is possible that women while considering dysuria to be "not normal" may associate it with a urinary tract infection rather than an STI. Men who are more likely than women to have dysuria (62\% versus $25 \%$ ) may realise its significance as an STI symptom and take protective measures. With only a small number of symptomatic women in our study having dysuria it is hard to draw any definite conclusions.

In men, the presence of urethral discharge did not significantly alter coital activity or condom use. Urethral discharge is associated with increased condom use but did not reach statistical significance in our analysis (OR 2.04, 95\% CI 0.87 to 4.78 ). Dysuria was associated with increased likelihood of condom use but had no effect on coital activity. These findings are consistent with those reported by Irwin et al in that factors associated with sexual activity while symptomatic include duration of symptoms $<7$ days, black race, and male sex. ${ }^{4}$ In our study, $68 \%$ of the men sought care within 7 days of symptom onset therefore reducing the observable time to notice any changes in sexual activity. It is possible that men who delay seeking care while symptomatic may be more likely to alter sexual behaviours. However, other studies suggest that longer symptom duration ( $>7$ days) was associated with increased likelihood of sexual activity while symptomatic in men and women. ${ }^{62}$

This study also draws attention to the fact that women and men frequently increase condom use in response to STI symptoms. Assuming that genitourinary symptoms are a result of an STI, it is likely that people will report a higher level of condom use when asked at the time of the healthcare visit. Numerous studies have shown the failure of condoms to prevent STI, which may be a combination of reporting bias, improper condom use, and possibly increased condom use after establishment of the infection.

Further research is needed to understand the reasons why some symptomatic women and men who may know themselves to be infected continue to engage in often unprotected coital activity. It is possible that simple awareness of what is normal and abnormal can be learned and would result in appropriate modification of sexual behaviours among symptomatic people.

STI prevention programmes need to focus in particular on men who may be more likely to increase STI transmission by not altering their coital activity even in the presence of fairly specific STI symptoms such as urethral discharge and dysuria. Men who have urethral discharge and continue sexual activity but do not use condoms, do not understand STI transmission, fail to recognise the significance of the symptoms, or lack concern for their partner's health. It also underscores the importance to develop means of reducing disease transmission that are more under the control of women.
Key messages

- Gender affects the way women and men alter sexual behaviours in response to genital symptoms

- It is possible that racial origin also impacts on sexual behaviours and further studies are needed to explore these complex influences

- STI prevention programmes need to better understand gender and racial differences in sexual behaviours

\section{Limitations}

Our study used self reported record of coital activity and condom use, hence reporting may not be completely reliable or accurate. We tried to improve the accuracy by using calendar recall; however, recall limitations and social desirability and reporting bias still exist. In addition, reporting of symptoms may not accurately reflect the respondent's variable perceptions and experience of normal and abnormal symptoms. This may be especially true for women who may have a harder time than men discerning normal from abnormal thus leading to over-reporting or under-reporting of symptoms. Our study population was recruited from an STD clinic and thus is a younger cohort of higher risk individuals than the general population. Therefore, the results may not be generalisable to older populations, to those seeking care in non-STD healthcare settings or to those not seeking care at all. In our regression models we looked at the sexual behaviours of only symptomatic individuals and the small sample size must be taken into consideration when drawing any conclusions based on our results. However, based on the exploratory nature of this study we think that our findings warrant additional studies to explore the complexities of sexual behaviours in symptomatic individuals. This study did not address the effect of symptom severity on coital activity or condom use. Respondents were not asked about the length of their relationships or the type of sexual partner (spouse or long term versus short term). Both of these factors have been shown to affect the likelihood of coital activity and condom use. A possible limitation of our study may be the sample bias introduced by recruitment of partners. However, there was no difference between index subjects and partners in symptoms or care seeking duration. We also did not find a predominance of partners in the asymptomatic group.

\section{CONCLUSIONS}

Our findings on gender differences in response to genitourinary symptoms show that black women alter sexual and condom use behaviours more appropriately than non-black women. Men on the other hand are less likely to alter their sexual activity. Changes in sexual behaviours may alter the risk of STI transmission independent of interactions with the healthcare system. Specifically targeting STI prevention programmes to better address these gender differences is crucial in developing strategies to reduce transmission. STI education and awareness programmes need to have gender specific interventions to be effective in motivating and maintaining STI preventive behaviours.

\section{ACKNOWLEDGEMENTS}

The authors would like to thank Tim Breen for excellent assistance in preparing the data for analysis. This work was supported by Public Health Service grant AI31494 from the National Institute of Allergy and Infectious Diseases, and by grant UR3/CCU5516481 from the Centers for Disease Control and Prevention. The work of Wanzhu Tu was supported in part by grant ROI HD042402 from the National Institute of Child Health and Human Development. 


\section{CONTRIBUTORS}

$\mathrm{AK}, \mathrm{DF}, \mathrm{DO}$, and $\mathrm{BB}$ contributed to the project design, development, and writing/editing of the manuscript; AK, HT, and WT contributed to the statistical and data analysis in the manuscript.

\section{Authors' affiliations}

A Khan, B E Batteiger, Division of Infectious Diseases, Indiana University School of Medicine, Indianapolis, IN, USA

J D Fortenberry, D P Orr, Division of Adolescent Medicine, Indiana University School of Medicine, Indianapolis, IN, USA

M' H Temkit, W Tu, Division of Biostatistics, Indiana University School of Medicine, Indianapolis, IN, USA

\section{REFERENCES}

1 Pescosolido BA. Beyond rational choice: the social dynamics of how people seek help. Am J Sociol 1992;97:1096-138.

2 Kramer MA, Aral SO, Curran JW. Self-reported behavior patterns of patients attending a sexually transmitted disease clinic. Am J Public Health 1980;70:997-1000

3 Leenaars PEM, Rombouts R, Kok G. Seeking medical care for a sexually transmitted disease: determinants of delay-behavior. Psychol Health 1993;8:17-32.

4 Irwin DE, Thomas JC, Spitters CE, et al. Self Reported sexual activity and condom use among symptomatic clients attending STD clinics. Sex Transm Dis 1999;26:286-90.

5 Anderson RM. The transmission dynamics of sexually transmitted diseases: the behavioral component. In: Wasserheit JN, Aral SO, Holmes KK, eds. Research issues in human behavior and sexually transmitted diseases in the AIDS era. Washington, DC: American Society for Microbiology, 1991:38-60.

6 Moses S, Ngugi EN, Bradley JE, et al. Health care-seeking behavior related to the transmission of sexually transmitted diseases in Kenya. Am J Public Health 1994;84:1947-51.

7 Darrow WW. Veneral infections in three ethnic groups in Sacramento. Am J Public Health 1976;66:446-50.

8 Wingood GM, DiClemente RJ. Cultural, gender, and psychosocial influences on HIV-related behaviors of African-American female adolescents: implications for the development of tailored prevention programs. Ethnicity and Disease 1992;2:381-8.
9 Darroch J, Myers L, Cassell J. Sex differences in the experience of testing positive for genital chlamydia infection: a qualitative study with implications for public health and for national screening programme. Sex Transm Infect 2003;79:372-4.

10 Leigh BC, Gillmore MR, Morrison DM. Comparison of diary and recall measure for recording alcohol consumption and sexual activity. J Clin Epidemiol 1998;51:119-27.

11 Hornsby PP, Wilcox AJ. Validity of questionnaire information on frequency of coitus. Am J Epidemiol 1989;130:94-9.

12 Lemmens R, Knibbe RA, Tan ES. Weekly recall and diary estimates of alcohol consumption in a general population survey. J Stud Alcohol 1988;49:131-5.

13 Kengne $\mathbf{P}$, Veas $F$, Vidal $N$, et al. Trichomonas vaginalis: repeated DNA target for highly sensitive and specific Polymerase chain reaction diagnosis. Cell Mol Biol 1994;40:813-31.

14 Aral SO, Mosher WD, Cates W. Vaginal douching among women of reproductive age in the United States: 1988. Am J Public Health 1992;82:210-4.

15 Evans B, Kell PD, Bond RA, et al. Racial origin, sexual lifestyle, and genital infection among women attending a genitourinary medicine clinic in London (1992). Sex Transm Infect 1998;74:45-9.

16 Warszawski J, Meyer L. Gender differences in persistent at risk sexual behaviors after a diagnosed sexually transmitted disease. Sex Transm Dis 1998;25:437-42

17 Wingood GM, DiClemente RJ. Partner influences and gender-related factors associated with noncondom use among young adult African American women. Am J Community Psychol 1998;26:29-51.

18 Williams M, Ross MW, Bowen AM, et al. An investigation of condom use by frequency of sex. Sex Transm Infect 2001;77:433-5.

19 Macaluso M, Demand MJ, Artz LM, et al. Partner type and condom use. AIDS 2000;14:537-46

20 Fortenberry JD, Wanzhu T, Harzelak J, et al. Condom use as a function of time in new and established adolescent sexual relationships. Am J Public Health 2002:92:211-13.

21 Zenilman JM, Weisman CS, Rompalo AM, et al. Condom use to prevent incident STDs: the validity of self-reported condom use. Sex Transm Dis 1995;22:15-21.

22 Upchurch DM, Brady WE, Reichart CA, et al. Behavioral contributions to acquisition of gonorrhea in patients attending an inner city sexually transmitted diseases clinic. J Infect Dis 1990;161:938-41.

23 O'Donnell L, San Doval A, Vornfett R, et al. STD prevention and the challenge of gender and cultural diversity: knowledge, attitudes, and risk behaviors among black and Hispanic inner-city STD clinic patients. Sex Transm Dis 1994:21:137-48. 